\title{
New Andreev-Type States in Superconducting Nanowires
}

\author{
A. A. Shanenko, ${ }^{1,3}$ M. D. Croitoru, ${ }^{2}$ R. G. Mints, ${ }^{4}$ and F. M. Peeters ${ }^{1}$ \\ ${ }^{1}$ TGM, Departement Fysica, Universiteit Antwerpen, Groenenborgerlaan 171, B-2020 Antwerpen, Belgium \\ ${ }^{2}$ EMAT, Departement Fysica, Universiteit Antwerpen, Groenenborgerlaan 171, B-2020 Antwerpen, Belgium \\ ${ }^{3}$ Bogoliubov Laboratory of Theoretical Physics, Joint Institute for Nuclear Research, 141980 Dubna, Russia \\ ${ }^{4}$ School of Physics and Astronomy, Raymond and Beverly Sackler Faculty of Exact Sciences, Tel Aviv University, Tel Aviv 69978, Israel
}

(Received 6 April 2007; published 10 August 2007)

\begin{abstract}
Superconducting nanowires can exhibit a spatially inhomogeneous pair condensate that leads to the formation of new Andreev-type states. Such states are mainly located beyond the regions where the order parameter is enhanced, and no normal-superconducting contact or external magnetic field is needed for their formation. Our numerical self-consistent solutions of the Bogoliubov-de Gennes equations for cylindrical nanowires, in the clean limit, demonstrate that these new Andreev-type states decrease the ratio of the energy gap to the critical temperature as compared to its bulk value. The low-lying excitations in a clean superconducting nanowire are these new Andreev-type states induced by quantum confinement of the electrons in the transverse direction.
\end{abstract}

DOI: 10.1103/PhysRevLett.99.067007

Recent advances in nanofabrication technology resulted in nanoscale structures like wires with width about $\sim 10 \mathrm{~nm}[1,2]$ and films with thickness of a few monolayers [3-5]. In particular, superconducting metallic nanowires or nanofilms are now attainable with electron mean free path being about the specimen width or thickness $[1,4]$. To a great extent such nanoscale superconducting specimens can be considered as being in the clean limit. In this case nonmagnetic impurities can only influence the electron motion parallel to the wire or film. Then, arguments similar to the Anderson theorem [6] make it possible to expect that the longitudinal scattering of electrons on such nonmagnetic imperfections cannot have a significant effect on the superconducting characteristics in the clean limit. The physical properties of a clean nanosuperconductor are governed by quantum confinement (QC) of electrons, which open up a unique and promising possibility of investigating the interplay between QC and superconductivity.

It is well established that the superconducting order parameter $\Delta(\mathbf{r})$ can be treated as a wave function of the center-of-mass motion of Cooper pairs (see the textbook [7] ). Hence, $\Delta(\mathbf{r})$ should vary with position in the presence of QC. The spatial variations of $\Delta(\mathbf{r})$ are expected to be especially significant for nanoscale specimens. Recently, such variations were calculated for a cylindrical nanowire $[8,9]$ and for a nanofilm [10] by numerically solving the Bogoliubov-de Gennes (BdG) equations. It was shown that the superconducting order parameter and its spatial inhomogeneity are strongly enhanced when a size/shapedependent superconducting resonance appears $[9,10]$. Such resonances result in a size-dependent increase of the critical temperature $T_{c}$ which was recently observed in aluminum and tin nanowires [1,2]. Therefore, a remarkable consequence of $\mathrm{QC}$ is the appearance of the size/
PACS numbers: 74.78.Na, 74.20.De, 74.45.+c, 74.81.Fa

shape-resonant superconductivity characterized by a nonuniform spatial distribution of the pair condensate.

Spatial variations of $\Delta(\mathbf{r})$ can result in Andreev reflection and quantization of low-energy excitations [11]. Under certain conditions, the excitations located in the areas of low values of $|\Delta(\mathbf{r})|$ cannot penetrate into the areas of higher values of $|\Delta(\mathbf{r})|$. This mechanism [Andreev mechanism (AM)] leads to Andreev quantization as previously discussed for a normal region of the intermediate state [11] and for a single Abrikosov vortex core [12]. In both cases the boundary conditions are specified by the bulk surroundings, i.e., $\Delta(\mathbf{r})$ approaches its bulk value $\Delta_{\text {bulk }}$ when $|\mathbf{r}| \rightarrow \infty$.

The situation is dramatically different in the presence of QC. An interesting interplay between AM and QC was recently reported [13] for a clean mesoscopic specimen with an embedded superconductor-normal-metal-superconductor structure. In this case QC plays a secondary role (as compared to AM) and results in oscillations of the Andreev levels as function of the specimen dimensions.

In the present study we investigate, for the first time, AM in a clean nanosized superconductor governed by QC. Based on a numerical self-consistent solution of the BdG equations for a cylindrical nanowire in the absence of a magnetic field, we show that on the nanoscale, AM manifests itself through the formation of new Andreev-type states mainly located beyond the regions where the order parameter is enhanced. Stress that these states cannot be localized in the areas where $\Delta(\mathbf{r})$ is suppressed, because the typical length scale for spatial variations of the order parameter is about the Fermi wavelength $\lambda_{F}\left(\lambda_{F} \ll \xi\right.$, where $\xi$ is the coherence length). In general, one may expect that the lowest quasiparticle states in a clean nanoscale superconducting specimen are always such new Andreev-type states. 
In the absence of magnetic field, $\Delta(\mathbf{r})$ can be taken real and the $\mathrm{BdG}$ equations yield [6]

$$
\begin{aligned}
& E_{i} u_{i}(\mathbf{r})=\left(-\frac{\hbar^{2}}{2 m_{e}} \nabla^{2}-\mu\right) u_{i}(\mathbf{r})+\Delta(\mathbf{r}) v_{i}(\mathbf{r}), \\
& E_{i} v_{i}(\mathbf{r})=\Delta(\mathbf{r}) u_{i}(\mathbf{r})-\left(-\frac{\hbar^{2}}{2 m_{e}} \nabla^{2}-\mu\right) v_{i}(\mathbf{r}),
\end{aligned}
$$

where $E_{i}$ is the energy of the excitations, $\mu$ is the chemical potential, and $m_{e}$ is the electron band mass. Next, we have to add the self-consistency relation

$$
\Delta(\mathbf{r})=g \sum_{i} u_{i}(\mathbf{r}) v_{i}^{*}(\mathbf{r})\left[1-2 f_{i}\right]
$$

In the above equation we denote the coupling constant as $g$, and $f_{i}=f\left(E_{i}\right)$ is the Fermi function. The summation in Eq. (3) is over the Debye window of the single-electron states, i.e., the interval from $\mu-\hbar \omega_{D}$ to $\mu+\hbar \omega_{D}$, where $\omega_{D}$ is the Debye frequency [6]. Finally, the chemical potential is determined from

$$
n=\frac{2}{V} \sum_{i} \int d^{3} r\left[\left|u_{i}(\mathbf{r})\right|^{2} f_{i}+\left|v_{i}(\mathbf{r})\right|^{2}\left(1-f_{i}\right)\right]
$$

where $n$ is the mean electron density and $V=\pi R^{2} L$ is the system volume.

We write the transverse boundary conditions on the nanowire surface as $\left.u_{i}(\mathbf{r})\right|_{\mathbf{r} \in S}=\left.v_{i}(\mathbf{r})\right|_{\mathbf{r} \in S}=0$ and take periodic boundary conditions in the longitudinal direction. For the chosen geometry we have $\Delta(\mathbf{r})=\Delta(\rho)$, where $\rho$, $\varphi, z$ are the cylindrical coordinates, and

$$
u_{i}(\mathbf{r})=u_{i}(\rho) \frac{e^{i m \varphi}}{\sqrt{2 \pi}} \frac{e^{i k z}}{\sqrt{L}}, \quad v_{i}(\mathbf{r})=v_{i}(\rho) \frac{e^{i m \varphi}}{\sqrt{2 \pi}} \frac{e^{i k z}}{\sqrt{L}},
$$

where $i=\{j, m, k\}, j$ is the quantum number associated with the transverse coordinate [the number of zeros of $u_{i}(\rho)$ and $v_{i}(\rho)$ for $\left.\rho<R\right], m$ is the azimuthal quantum number, and $k$ is the wave vector of the quasifree electron motion parallel to the nanowire. We remark that the terms including $\Delta(\mathbf{r})$ are local in Eqs. (1) and (2), which follows from the delta-function approximation for the electronelectron effective interaction (see textbooks [6,7]). Nonlocality may result in a smearing of the oscillatory behavior of the superconducting order parameter. However, this smearing will not be significant in our nanowires because the transverse profile of the order parameter is dictated by QC.

As mentioned above, superconducting properties of a sample depend on the number of single-electron states in the energy interval from $\mu-\hbar \omega_{D}$ to $\mu+\hbar \omega_{D}$. This number varies with the radius of the nanowire. In a clean metallic nanowire QC splits the band of single-electron states in a series of one-dimensional subbands. An increase of the radius of the nanowire leads to a decrease of the energy of the subbands. When the bottom of a certain subband passes through the Fermi level, the number of relevant single-electron states increases abruptly. This abrupt increase results in a series of radius-dependent resonances $[9,10]$. At the resonant values of the nanowire radius the order parameter is strongly enhanced as compared to its bulk value and exhibits a nonuniform spatial distribution. The smaller the radius of the nanowire, the stronger the resonance $[9,10]$.

In Fig. 1(a) we show $\Delta(\rho)$ as obtained from a numerical self-consistent solution of Eqs. (1) and (2) for an aluminum nanowire with $R=0.87 \mathrm{~nm}$ (one of the resonant radii) at zero temperature. The values of the parameters used in the calculations are $\hbar \omega_{D}=32.31 \mathrm{meV}, \quad g N(0)=0.18$, $\mu_{\text {bulk }}=0.9 \mathrm{eV}$, where $N(0)$ is the bulk density of states at the Fermi level and $\mu_{\text {bulk }}$ is the bulk chemical potential (the Fermi level) (see, for details, discussion about an effective Fermi level in Ref. [10]). The BCS coherence length for the chosen parameters is $\xi_{0}=1.6 \mu \mathrm{m}$ [7]. It follows from Fig. 1(a) that at $R=0.87 \mathrm{~nm}$ we have a giant enhancement of the superconducting order parameter $\left(\Delta_{\text {bulk }}=0.25 \mathrm{meV}\right)$, and, at the same time, $\Delta(\rho)$ is spatially dependent. In this case there are five relevant quasiparticle subbands with quantum numbers $j=0, m=0$, $j=0,|m|=1$ and $j=0,|m|=2$. It is worth noting that only the low-lying states contribute to $\Delta(\rho)$. The others are excluded because the corresponding single-electron ener-

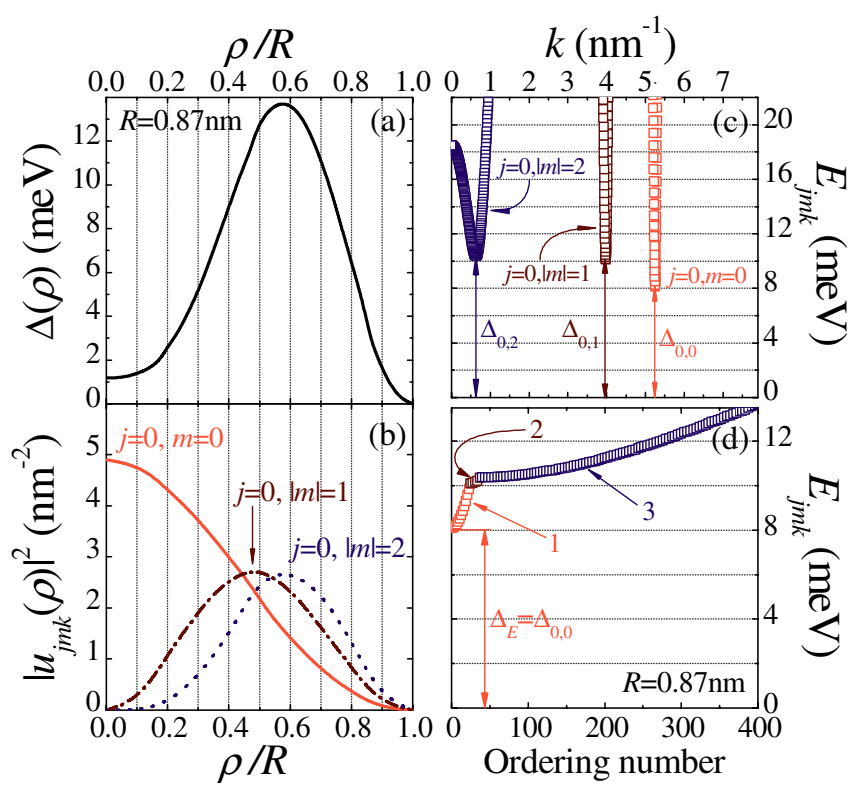

FIG. 1 (color online). The resonant point $R=0.87 \mathrm{~nm}$ : (a) the superconducting order parameter $\Delta(\rho)$, (b) the square of the absolute value of the transverse particlelike wave function $u_{j m k}(\rho)$ for the relevant quasiparticle branches (the lowest state is presented for any given quasiparticle branch), (c) the excitations energies $(\square)$ as function of $k$ for the lowest quasiparticle branches together with the branch gaps $\Delta_{j m}$, and (d) the quasiparticle energies ordered in the ascending manner vs the ordering number together with the total energy gap $\Delta_{E}$. 
gies do not belong to the interval from $\mu-\hbar \omega_{D}$ to $\mu+$ $\hbar \omega_{D}$. The dominant contribution to the superconducting order parameter comes from the two branches with $j=0$, $m=-2$ and $j=0, m=2$ that determine the giant enhancement of the order parameter at $\rho=0.58 R$.

The probability densities to locate electron-type and hole-type quasiparticles along the transverse coordinate $\rho$ are given by $\left|u_{j m k}(\rho)\right|^{2}$ and $\left|v_{j m k}(\rho)\right|^{2}$, respectively. Our numerical analysis shows that $u_{j m k}(\rho)$ and $v_{j m k}(\rho)$ are almost proportional to each other. It follows from Fig. 1(b) that the profile of $\left|u_{j k m}(\rho)\right|^{2}$ at $j=0,|m|=2$ is similar to the profile of $\Delta(\rho)$ in the region where $\Delta(\rho)$ is enhanced. The contribution to $\Delta(\rho)$ from the quasiparticle states with $j=0,|m|=1$ and $j=0, m=0$ is minor, and these states are mainly located outside the area of enhanced order parameter. We refer to them as the new Andreev-type states induced by QC.

In Fig. 1(c) the quasiparticle energies are plotted as function of the wave vector $k$ for the five relevant branches. For any quasiparticle branch specified by $j$ and $m$ we can introduce an energy gap $\Delta_{j m}$, the minimal quasiparticle energy. In the absence of superconductivity the particlelike and holelike excitations are decoupled and, so, $\Delta_{j m}=0$. For the superconducting state we get $\Delta_{j m} \neq 0$, and one can define the total energy gap $\Delta_{E}=\min \Delta_{j m}$. As seen, $\Delta_{0,2}$ and $\Delta_{0,-2}$, corresponding to the branches forming the profile of the enhancement in the order parameter, are larger than $\Delta_{0,0}, \Delta_{0,1}$, and $\Delta_{0,-1}$ associated with the new Andreev-type states. The energy density of the quasiparticle states with $j=0,|m|=2$ is much higher than that of the Andreev-type levels with $j=0,|m|=1$ and $j=0$, $m=0$. This explains why the contribution of the states with $j=0,|m|=2$ to the superconducting properties is the most important. We remark that these states have sufficiently long wavelengths $(\approx 10-20 \mathrm{~nm}$ ) in the direction parallel to the nanowire. Hence, one can expect that the profile of the order parameter will not be strongly influenced by surface roughness and, therefore, the new Andreev-type states will only be weakly altered. In Fig. 1(d) the quasiparticle energies ordered in ascending manner are plotted versus the ordering number. One can distinguish three regimes in Fig. 1(d): (1) only the quasiparticle states with $j=0, m=0$ contribute to the spectrum; (2) the states with $j=0,|m|=1$ appear in addition to those with $j=0, m=0$; (3) the states with $j=0$, $|m|=2$ dominate. The lowest branch corresponds to $j=$ $0, m=0$ (so that $\Delta_{E}=\Delta_{0,0}=8.1 \mathrm{meV}$ ), and exactly these quasiparticles are the most remote from the area of giant spatial variation in $\Delta(\rho)$ at $\rho / R=0.58$. As seen from Fig. 1(d), any time when a new quasiparticle branch starts to contribute to the excitation spectrum, we observe a kink in the dependence of the quasiparticle energy on the ordering number.

In order to demonstrate a more complex example, in Fig. 2(a) $\Delta(\rho)$ is plotted for another resonant value $R=$ $0.97 \mathrm{~nm}$ (the rest of the input parameters are the same as

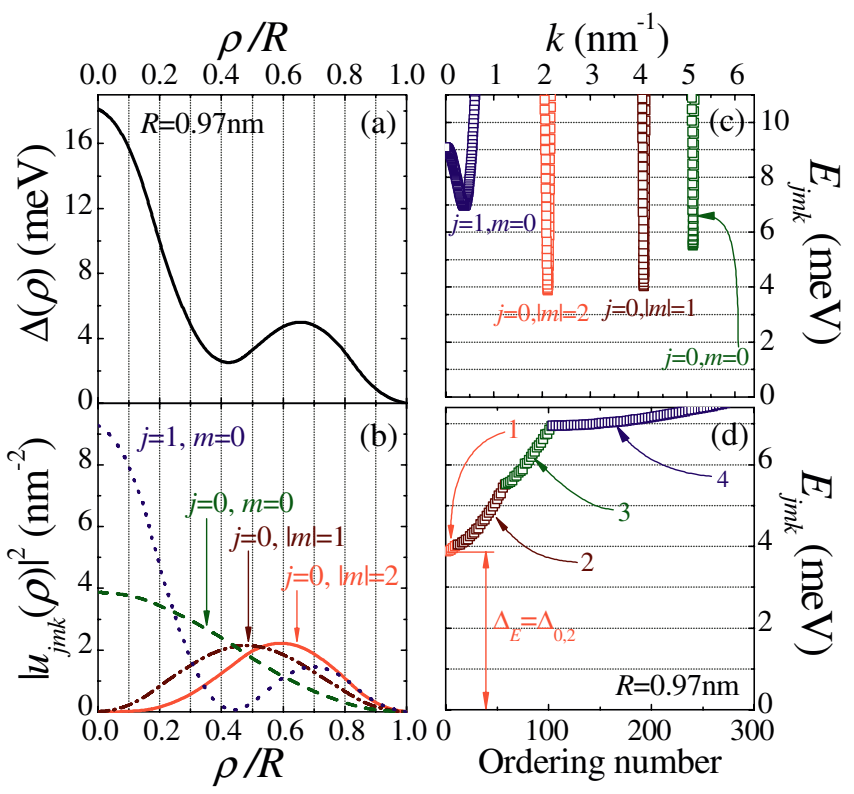

FIG. 2 (color online). The same as Fig. 1 but now for the resonant point $R=0.97 \mathrm{~nm}$.

for Fig. 1). In this example there are six quasiparticle branches contributing to the superconducting properties. In addition to the five branches mentioned above, there is a new one with $j=1, m=0$ which strongly affects the profile of $\Delta(\rho)$. The probability density $\left|u_{j m k}(\rho)\right|^{2}$ is shown in Fig. 2(b). It is seen from the data that excitations with $j=0,|m|=1$ and $j=0,|m|=2$ are located far apart from the region of the enhancement in $\Delta(\rho)$ at $\rho=0$. These states form the four new Andreev-type quasiparticle branches with the lowest branch gaps $\Delta_{0,2}=3.87 \mathrm{meV}$ and $\Delta_{0,1}=4.04 \mathrm{meV}$, as seen from Fig. 2(c) and 2(d). The interplay between AM and QC is now more complicated due to the presence of the two local maxima in $\Delta(\rho)$. Hence, in addition to the states with $j=0,|m|=1$ and $j=0,|m|=2$, there are the Andreev-type states with $j=$ $0, m=0$ that try "to avoid" the second, less pronounced maximum of $\Delta(\rho)$ situated at $\rho / R=0.68$. As expected, the branch gap is now higher, $\Delta_{0,0}=5.51 \mathrm{meV}$. Thus, at $R=0.97$ we have the four different branch gaps and, so, four regimes appear in the quasiparticle energy ordered in the ascending manner and plotted versus the ordering number in Fig. 1(d). The resonance shown in Fig. 2 completely decays when the radius increases up to $R \approx$ $1.1 \mathrm{~nm}$. For $R=1.1 \mathrm{~nm}$ the spatially averaged order parameter is only about $0.2 \mathrm{meV}$, and no significant effect of $\mathrm{AM}$ is found. In particular, the difference between $\Delta_{0,2}$ and $\Delta_{1,0}$ is here much less pronounced, within $10 \%$ [compare with Fig. 2(c)].

An important consequence of $\mathrm{AM}$ is a modification of the ratio of the zero-temperature gap to the critical temperature. In Fig. 3 we plot $\Delta_{E} / k_{B} T_{c}$ calculated for an aluminum cylindrical nanowire as a function of $R$. At a resonant point $\mathrm{AM}$ significantly reduces the energy gap but has little effect on $T_{c}$. As a result the ratio $\Delta_{E} / k_{B} T_{c}$ 


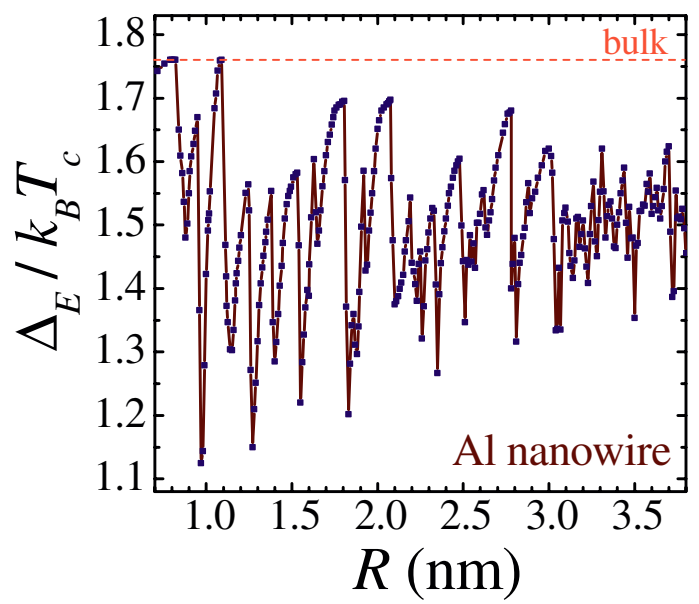

FIG. 3 (color online). The ratio $\Delta_{E} / k_{B} T_{c}$ vs the radius of a cylindrical aluminum nanowire.

decreases with respect to the well-known bulk BCS value $\Delta_{\text {bulk }} / k_{B} T_{c \text {,bulk }}=1.76$ typical for a spatially uniform pair condensate. Between the points of the width-dependent superconducting resonances, where AM is less important, $\Delta_{E} / k_{B} T_{c}$ increases: the more profound the drop of the superconducting characteristics, the larger the increase. Note that the width-dependent ratio of the gap to the critical temperature never exceeds the uniform (bulk) limit 1.76. Moreover, only in few points the curve in Fig. 3 approaches this limiting value. It occurs when the superconducting order parameter is strongly reduced and, so, nearly uniform in space. Oscillations of $\Delta_{E} / k_{B} T_{c}$ slowly decay when the nanowire radius increases (due to weakening of the width-dependent resonances, see Ref. [9] ). At $R>10 \mathrm{~nm}$ the ratio $\Delta_{E} / k_{B} T_{c}$ is very close to the bulk value 1.76. In the previous paragraphs we considered, for the sake of simplicity, ultrathin nanowires (with $R \approx$ $1 \mathrm{~nm}$ ) which may exhibit a finite resistance originating from thermal and, possibly, quantum fluctuations (see, for instance, Refs. [1,2]). In the case of $R \approx 3-5 \mathrm{~nm}$, corresponding to the narrowest superconducting nanowires fabricated in recent experiments [1,2], the size superconducting resonances are less profound as compared to $R \approx$ $1 \mathrm{~nm}$. However, they are noticeable, and the new Andreevtype states are still of importance. In particular, as seen from Fig. 3, the ratio $\Delta_{E} / k_{B} T_{c}$ for $R \approx 4 \mathrm{~nm}$ still differs about $10 \%$ from the bulk value.

To conclude, quantum confinement is the major effect governing the superconducting properties of a clean nanoscale specimen. This results in size- or shape-dependent superconducting resonances with a nonuniform spatial distribution of the order parameter. Such inhomogeneity in the order parameter leads to the formation of new Andreev-type states trying "to escape" the domains of enhanced order parameter and being mainly located outside such domains. These new Andreev-type states result in a decrease of the ratio of the energy gap to the critical temperature as compared to bulk. A numerical selfconsistent solution of the Bogoliubov-de Gennes equations for a cylindrical metallic nanowire demonstrates that this decrease is significant for nanowires with diameters less than $\sim 10 \mathrm{~nm}$. We recall the reader that the ordinary Andreev bound states appearing in the core of an isolated vortex almost "kill" the gap in the excitation spectrum [12]. On the contrary, the new Andreev-type states can only somewhat reduce the energy gap. This is due to the fact that AM plays a secondary role as compared to QC in the case of interest. We expect that the low-lying excitations in a clean nanosuperconductor are such new Andreev-type states which can be probed, e.g., through tunneling experiments.

The authors thank D. Y. Vodolazov for helpful remarks. This work was supported by the Flemish Science Foundation (FWO-VI), the Belgian Science Policy, and BOF-TOP (University of Antwerpen).

[1] M. Savolainen, V. Touboltsev, P. Koppinen, K.-P. Riikonen, and K. Arutyunov, Appl. Phys. A 79, 1769 (2004); M. Zgirski, K.-P. Riikonen, V. Touboltsev, and K. Arutyunov, Nano Lett. 5, 1029 (2005).

[2] M. L. Tian, J. G. Wang, J. S. Kurtz, Y. Liu, M. H. W. Chan, T. S. Mayer, and T.E. Mallouk, Phys. Rev. B 71, 104521 (2005).

[3] Y. Guo, Y.-F. Zhang, X.-Y. Bao, T.-Z. Han, Z. Tang, L.-X. Zhang, W.-G. Zhu, E. G. Wang, Q. Niu, Z. Q. Qiu, J.-F. Jia, Z.-X. Zhao, and Q. K. Xue, Science 306, 1915 (2004).

[4] M. M. Özer, J. R. Thompson, and H. Weitering, Nature Phys. 2, 173 (2006); Phys. Rev. B 74, 235427 (2006).

[5] D. Eom, S. Qin, M.-Y. Chou, and C. K. Shih, Phys. Rev. Lett. 96, 027005 (2006).

[6] P. G. de Gennes, Superconductivity of Metals and Alloys (Benjamin, New York, 1966).

[7] A. L. Fetter and J. D. Walecka, Quantum Theory of ManyParticle Systems (Dover, New York, 2003).

[8] J.E. Han and V.H. Crespi, Phys. Rev. B 69, 214526 (2004).

[9] A. A. Shanenko, M. D. Croitoru, M. Zgirski, F. M. Peeters, and K. Arutyunov, Phys. Rev. B 74, 052502 (2006).

[10] A. A. Shanenko, M. D. Croitoru, and F. M. Peeters, Phys. Rev. B 75, 014519 (2007).

[11] A. F. Andreev, Sov. Phys. JETP 22, 455 (1966).

[12] C. Caroli, P. G. de Gennes, and J. Matricon, Phys. Lett. 9, 307 (1964); R. G. Mints and A. L. Rachmanov, Solid State Commun. 16, 747 (1975).

[13] N. B. Kopnin, A. S. Mel'nikov, V. I. Pozdnyakova, D. A. Ryzhov, I. A. Shereshevskii, and V. M. Vinokur, Phys. Rev. Lett. 95, 197002 (2005). 